\title{
1 Effective Cell Membrane Tension is Independent of Substrate
}

3 Eva Kreysing ${ }^{1, *}$, Jeffrey Mc Hugh ${ }^{2,3, *}$, Sarah K. Foster ${ }^{1,4}$, Kurt Andresen ${ }^{5}$, Ryan D. Greenhalgh ${ }^{1}$,

4 Eva K. Pillai ${ }^{1}$, Andrea Dimitracopoulos ${ }^{1}$, Ulrich F. Keyser ${ }^{2, \#,}$ Kristian Franze ${ }^{1,6,7, \#}$

6 1) Department of Physiology, Development and Neuroscience, University of Cambridge, $7 \quad$ Cambridge CB2 3DY, UK

8 2) Biological and Soft Systems, Maxwell Centre, Cavendish Laboratory, Cambridge CB3 OHE, UK

9 3) Neuroglial Interactions in Cerebral Physiopathology, CIRB, CNRS UMR 7241/INSERM U1050, 10 Collège de France, Paris 75005, France

11 4) Systems Biology of Microbial Communities, Cluster of Excellence - CMFI, University of 12 Tübingen, 72076 Tübingen, Germany

13 5) Department of Physics, Gettysburg College, Gettysburg, PA 17325, United States of America

14 6) Institute of Medical Physics, Friedrich-Alexander-Universität Erlangen-Nürnberg, 91052

15 Erlangen, Germany.

16 7) Max-Planck-Zentrum für Physik und Medizin, 91054 Erlangen, Germany

17

$18 *$ These authors contributed equally.

19\# To whom correspondence should be addressed: ufk20@cam.ac.uk or kf284@cam.ac.uk 


\section{Abstract}

22 Most animal cells are surrounded by a cell membrane and an underlying actomyosin cortex.

23 Both structures are linked with each other, and they are under tension. Membrane tension and

24 cortical tension both influence many cellular processes, including cell migration, division, and

25 endocytosis. However, while actomyosin tension is regulated by substrate stiffness, how

26 membrane tension responds to mechanical substrate properties is currently poorly understood.

27 Here, we probed the effective membrane tension of neurons and fibroblasts cultured on glass

28 and polyacrylamide substrates of varying stiffness using optical tweezers. In contrast to

29 actomyosin-based traction forces, both peak forces and steady state tether forces of cells

30 cultured on hydrogels were independent of substrate stiffness and did not change after blocking

31 myosin II activity using blebbistatin, indicating that tether and traction forces are not directly

32 linked with each other. Peak forces on hydrogels were about twice as high in fibroblasts if

33 compared to neurons, indicating stronger membrane-cortex adhesion in fibroblasts. Finally,

34 tether forces were generally higher in cells cultured on hydrogels compared to cells cultured on

35 glass, which we attribute to substrate-dependent alterations of the actomyosin cortex and an

36 inverse relationship between tension along stress fibres and cortical tension. Our results provide

37 new insights into the complex regulation of membrane tension, and they pave the way for a

38 deeper understanding of biological processes instructed by it. 


\section{Introduction}

41 The cell membrane forms the interface between the interior of a cell and its environment. As a

42 lipid bilayer surrounded by aqueous solutions, cell membranes are usually under tension.

43 Different external forces are thought to contribute to membrane tension, including osmotic

44 pressure and interactions with the force generating cytoskeleton. The plasma membrane is

45 supported by the underlying actomyosin cortex, and molecular linkers form tight connections

46 between the two layers.

47 Many cellular processes, such as the regulation of cell morphology, migration, division, stem cell

48 fate choice, and endo- and exocytosis, are regulated by membrane tension ${ }^{1-6}$. Despite its

49 biological importance ${ }^{7-9}$, membrane tension is still difficult to quantify. In plane membrane

50 tension is defined as the force per unit length acting tangentially on the membrane. However,

51 because of the membrane's coupling to cytoskeletal elements, measurements of the 'effective'

52 membrane tension usually contain contributions of the underlying actomyosin cortex and other

53 force-generating elements, as membrane-cytoskeleton adhesion has to be overcome ${ }^{10,11}$.

54 The actomyosin cortex is coupled to the extracellular environment through integral membrane

55 proteins such as integrins and cadherins. It generates tensile forces which are exerted on the

56 environment. These forces, and hence cytoskeletal tension, increase with substrate stiffness

5712,13 , suggesting that the measured effective membrane tension should increase on stiffer

58 substrates. However, the regulation of membrane tension by substrate mechanics is currently

59 poorly understood.

60 


\section{Results and discussion}

62 We here measured the effective membrane tension of cells cultured on substrates of varying

63 stiffness by pulling thin membrane tethers from the cell using a membrane-adherent bead held

64 in an optical trap (OT) 2,14,15 (Fig. 1a, b; Fig. S1). Throughout a pull, the force on the tether can be

65 continuously monitored, resulting in a characteristic force curve (Fig. 1c). The force rises quickly

66 after initiation of the pull and reaches a peak before decreasing sharply. We attribute this peak

67 force (PF) to the initial local detachment of plasma membrane from the actomyosin cortex (Fig.

68 1a). Subsequently, the tether is pulled until it reaches a set length. During this elongation

69 process, the plasma membrane slides over the actin cortex while bonds between the membrane

70 and the lipid binding proteins located in or at the cortex are broken and quickly re-established ${ }^{16}$.

71 Following the elongation phase, the bead is held in a stable position and the steady-state force

72 (SSF) is measured (Fig. 1b). The SSF is thought to reflect a combination of the in-plane plasma

73 membrane tension and the membrane-cortex adhesion, which is in turn influenced by the

74 cortical tension ${ }^{16,17}$.

76 Tether forces are independent of hydrogel stiffness but PFs on glass depend on cell type

77 To test the effect of substrate mechanics and cortical tension on membrane tension, we

78 performed tether pulls in fibroblasts, which possess a fully developed actomyosin cortex, and in

79 neuronal axons, whose sub-membranous cytoskeleton is characterized by actin rings spaced by

80 spectrin tetramers. Cells were cultured on glass as well as on hydrogels within a stiffness range

81 adjusted to match their natural environment. For fibroblasts, hydrogel stiffnesses ranged from 
$82100 \mathrm{~Pa}$ (very soft; similar to subcutaneous adipose tissue), to 10kPa (stiff; already in the stiffness

83 range of many fibrotic tissues) ${ }^{18}$. For neurons, the highest substrate stiffness used was $1 \mathrm{kPa}$,

84 corresponding to the highest stiffness experienced by these cells in vivo ${ }^{19}$.

85 Peak forces were about twice as high in fibroblasts if compared to neuronal axons (Fig. 1e, h). In

86 both cell types, PFs were similar across all tested hydrogel substrates. In neurons, also PFs

87 measured in axons cultured on glass were similar to those seen on hydrogels (Fig. 1h). In

88 fibroblasts, however, we found significantly lower PFs when cultured on glass compared to those

89 cultured on hydrogels (Fig. 1e).

90 SSFs were significantly higher for fibroblasts and neurons cultured on hydrogels compared to

91 those cultured on glass (Fig. 1f, i). Similar to PFs, there was also no significant change in the SSFs

92 among the cells cultured on differently stiff hydrogels.

93 In contrast, actomyosin-based traction forces scale with substrate stiffnesses for both neurons

94 and fibroblasts 22 . Also in our culture conditions, cellular traction forces significantly increased on

95 stiffer substrates in both cell types within the investigated stiffness range (Fig. 1j, k), while tether

96 forces were independent of hydrogel substrate stiffness (Fig.1d-i). These data indicated that

97 traction forces and tether forces are not tightly coupled.

99 Tether forces on hydrogels but not SSFs on glass are independent of actomyosin contractility

100 Cellular cortical tension is mediated by actomyosin contractility ${ }^{20}$. In order to further examine

101 the interplay between cortical tension and membrane tension, we measured tether forces in 
102 fibroblasts cultured on glass and $10 \mathrm{kPa}$ hydrogels, where we would expect the highest cortical

103 tension ${ }^{22}$, following treatment with the myosin inhibitor blebbistatin.

104 Blebbistatin treatment had no effect on tether forces in fibroblasts cultured on hydrogels (Fig. 2).

105 While PFs also did not change significantly after blocking actomyosin contractility in fibroblasts

106 cultured on glass, SSFs increased on glass following blebbistatin treatment ${ }^{21}$ (Fig 2b).

\section{Discussion}

109 Here we found that the effective membrane tension, which is thought to scale with in-plane

110 membrane tension, does not change as a function of hydrogel substrate stiffness within a

111 physiologically relevant stiffness range in either fibroblasts or neurons (Fig. 1d-i). In contrast,

112 actomyosin-based traction forces increased on stiffer substrates (Fig. 1j-k), suggesting that

113 membrane tension is not linked to cellular contractility ${ }^{28}$. In line with this thought, the

114 application of the myosin II blocker, blebbistatin, did not alter the effective membrane tension in

115 cells cultured on hydrogels (Fig. 2).

116 However, we found differences in effective membrane tension between cells cultured on

117 hydrogels and glass. In fibroblasts, both PFs and SSFs were significantly lower in cells grown on

118 glass in comparison to those grown on hydrogels (Fig. 1d-f). On glass, the application of

119 blebbistatin led to a significant increase in effective membrane tension; both PFs and SSFs rose

120 to levels found in fibroblasts cultured on hydrogels (Fig. 2). In neurons, SSFs were also

121 significantly lower on glass than on hydrogels, whereas PFs on glass were similar to those found

122 on hydrogels of different stiffnesses (Fig. 1g-i). 


\section{Peak forces as a readout of membrane-cortex adhesion}

124 Our results suggest that the largest contribution to the measured PF is the initial force required

125 to detach the membrane from the cortex, which is strongly related to membrane-cortex

126 adhesion (in analogy to attempting to open Velcro from the middle). Fibroblasts have a fully

127 developed actomyosin cortex, and proteins such as ezrin/radixin/moesin (ERM) proteins can

128 connect membrane proteins to the actin cytoskeleton throughout the cell surface. Neuronal

129 axons, on the other hand, have a highly ordered, periodic actin-spectrin network underneath

130 their membrane 23 (Fig. 3b), and much less area for ERM proteins to connect the membrane to

131 the actin rings, suggesting that membrane-cortex adhesion in axons is very low. Hence, this

132 difference in the structure of the cell cortex may explain why PFs are about twice as high in

133 fibroblasts than in neuronal axons cultured on physiologically stiff hydrogels.

134 The cortical structure, and hence likely also membrane-cortex adhesion, of most cell types varies

135 depending on, for example, the mitotic state 24,20 or the cell spread area ${ }^{25}$. The actin

136 cytoskeleton in fibroblasts changes drastically between hydrogel and glass surfaces (Fig. S2).

137 Glass is orders of magnitude stiffer than hydrogels, and it has different surface properties. These

138 mechanical and chemical differences between the substrates may not only lead to changes in

139 the actin cytoskeleton but also cause changes in membrane-cortex adhesion, explaining why PFs

140 in fibroblasts are higher on hydrogels than on glass. In neurons, where the actin-spectrin

141 network is preserved on the different substrates and membrane-cortex adhesion is likely low in

142 either environment, PFs were similar on hydrogel and glass substrates.

\section{Interpretation of Steady State Forces}


144 The significantly lower SSFs on glass compared to hydrogels in both cell types could result from

145 changes in either the in-plane membrane tension and/or the adhesion between cortex and

146 plasma membrane. As depicted in Figure 3, these differences in SSFs could be explained by a

147 purely mechanical model ${ }^{26}$.

148 Particularly on very stiff glass substrates, fibroblasts developed strong stress fibres. Recent

149 experiments suggested that perturbations of stress fibres lead to an increase in tension of the

150 nearby cortex ${ }^{27}$. Thus, the contraction of stress fibres on glass might release tension in the cortex

151 and the membrane between focal adhesion sites (Fig. 3a), explaining why SSFs in fibroblasts

152 were lower on glass than on hydrogels (Fig. 1f), and why blebbistatin treatment, which relaxes

153 stress fibres, led to a significant increase in SSFs on glass but not on hydrogels (Fig. 2b).

154 However, blebbistatin treatment does not only affect stress fibres but also cortical tension. The

155 lack of a measurable change of SSFs after blebbistatin treatment in fibroblasts cultured on

156 hydrogels suggests either that the influence of the cortical tension on the SSFs is negligible, or

157 that the cortical tension changes very little under this treatment.

158 In developing neurons, grow cones at the leading tips of growing axons pull on their axons. The

159 magnitude of forces generated by growth cones increases with substrate stiffness (Fig. 1k),

160 suggesting that tension in the actin-spectrin cortex increases near the growth cone on stiffer

161 substrates and thus affects the plasma membrane tension in a similar way as described above

162 for fibroblasts (Fig. 3). 


\section{Conclusion}

165 We found that effective membrane tension of cells cultured on hydrogels is independent of

166 substrate stiffness and traction forces. This robustness suggests that membrane tension might

167 either not be influenced by cortical tension, or it is actively maintained within a certain range.

168 Another possibility is that traction forces transmitted to the cortex might dissipate quickly so

169 that overall cortical tension would not scale with local traction forces ${ }^{22}$. If cortical and

170 membrane tension are coupled, changes in membrane tension on the different hydrogels would

171 happen only locally ${ }^{29}$ near focal adhesions and cannot be seen when probed further away.

172 Future technological developments enabling highly resolved membrane tension measurements

173 will shed light on these important unresolved questions.

174

\section{Author Contributions}

176 J. Mc H., E. K., and K. A. performed tether pulling experiments. J. Mc H. analysed the results

177 from OT experiments. E. K. cultured 3 T3 fibroblasts and prepared hydrogels. S. K. F. dissected

178 and cultured Xenopus laevis RGCs and prepared hydrogels. A.D. performed TFM experiments

179 with 3 T3 fibroblasts. R. G. performed the TFM experiments with neurons and all TFM analyses. E.

180 K. and E. P. performed the immunostainings. J. Mc H. and E. K. designed experiments with 3 T3

181 fibroblasts. J. Mc H., S. K. F., K. A., and U. F. K. designed pulling experiments with X. laevis RGCs.

182 S. K. F., E. K., U.F.K. and K. F. conceived the project. E. K., J. Mc H., S. K. F., U.F.K. and K.F. wrote

183 the manuscript. 


\section{Acknowledgments}

185 The authors would like to thank Alex Winkel and Joy Thompson for AFM measurements of

186 hydrogels and Ewa Paluch, Ruby Peters and Aki Stubb for discussions. J. Mc H. acknowledges

187 funding from AFOSR (Grant No. FA9550-17-1-0118). S. F. K. acknowledges funding from the

188 Herchel Smith Foundation. U. F. K. was supported by ERC Consolidator Grant DesignerPores No.

189647144, K. F. was supported by the European Research Council (Consolidator Grant 772426) and

190 the Alexander von Humboldt Foundation (Alexander von Humboldt Professorship). 


\section{References}

193 1. Raucher, D. \& Sheetz, M. P. Cell Spreading and Lamellipodial Extension Rate Is Regulated by

194 Membrane Tension. J. Cell Biol. 148, 127-136 (2000).

195 2. Nussenzveig, H. M. Cell membrane biophysics with optical tweezers. Eur. Biophys. J. 47, 499-

196514 (2018).

197 3. Gauthier, N. C., Fardin, M. A., Roca-Cusachs, P. \& Sheetz, M. P. Temporary increase in plasma

198 membrane tension coordinates the activation of exocytosis and contraction during cell

199 spreading. Proc. Natl. Acad. Sci. U. S. A. 108, 14467-14472 (2011).

200 4. Keren, K. et al. Mechanism of shape determination in motile cells. Nature 453, 475-480

201 (2008)

202 5. De Belly, H. et al. Membrane Tension Gates ERK-Mediated Regulation of Pluripotent Cell Fate.

203 Cell Stem Cell 28, 273-284.e6 (2021).

204 6. Dai, J. \& Sheetz, M. P. Regulation of endocytosis, exocytosis, and shape by membrane tension.

205 Cold Spring Harb. Symp. Quant. Biol. 60, 567-571 (1995).

206 7. Sheetz, M. P. Cell control by membrane-cytoskeleton adhesion. Nat. Rev. Mol. Cell Biol. 2,

207 392-396 (2001).

208 8. Pontes, B., Monzo, P. \& Gauthier, N. C. Membrane tension: A challenging but universal

209 physical parameter in cell biology. Semin. Cell Dev. Biol. 71, 30-41 (2017).

210 9. Pontes, B. et al. Membrane tension controls adhesion positioning at the leading edge of cells.

211 J. Cell Biol. 216, 2959-2977 (2017).

212 10. Salbreux, G., Charras, G. \& Paluch, E. Actin cortex mechanics and cellular morphogenesis.

213 Trends Cell Biol. 22, 536-545 (2012). 
214 11. Clark, A. G., Wartlick, O., Salbreux, G. \& Paluch, E. K. Stresses at the Cell Surface during

215 Animal Cell Morphogenesis. Curr. Biol. 24, R484-R494 (2014).

216 12. Sabass, B., Gardel, M. L., Waterman, C. M. \& Schwarz, U. S. High Resolution Traction

217 Force Microscopy Based on Experimental and Computational Advances. Biophys. J. 94, 207-

$218220(2008)$

219 13. Califano, J. P. \& Reinhart-King, C. A. Substrate Stiffness and Cell Area Predict Cellular

220 Traction Stresses in Single Cells and Cells in Contact. Cell. Mol. Bioeng. 3, 68-75 (2010).

221 14. Sheetz, M. P. Cell control by membrane-cytoskeleton adhesion. Nat. Rev. Mol. Cell Biol.

$222 \quad 2,392-396(2001)$.

223 15. Datar, A., Bornschlögl, T., Bassereau, P., Prost, J. \& Pullarkat, P. A. Dynamics of membrane 224 tethers reveal novel aspects of cytoskeleton-membrane interactions in axons. Biophys. J. 108, 225 489-497 (2015).

226 16. Gauthier, N. C., Masters, T. A. \& Sheetz, M. P. Mechanical feedback between membrane 227 tension and dynamics. Trends Cell Biol. 22, 527-535 (2012).

228 17. Sens, P. \& Plastino, J. Membrane tension and cytoskeleton organization in cell motility. J. 229 Phys. Condens. Matter 27, (2015).

230 18. Wells, R. G. Tissue Mechanics and Fibrosis. Biochim. Biophys. Acta 1832, 884-890 (2013).

231 19. Franze, K., Janmey, P. A. \& Guck, J. Mechanics in Neuronal Development and Repair.

232 Annu. Rev. Biomed. Eng. 15, 227-251 (2013).

233 20. Chugh, P. et al. Actin cortex architecture regulates cell surface tension. Nat. Cell Biol. 19, 234 689-697 (2017). 
235 21. Lieber, A. D., Yehudai-Resheff, S., Barnhart, E. L., Theriot, J. A. \& Keren, K. Membrane

236 Tension in Rapidly Moving Cells Is Determined by Cytoskeletal Forces. Curr. Biol. 23, 1409-

$237 \quad 1417(2013)$

238 22. Rheinlaender, J. et al. Cortical cell stiffness is independent of substrate mechanics. Nat.

239 Mater. 19, 1019-1025 (2020).

240 23. Xu, K., Zhong, G. \& Zhuang, X. Actin, spectrin, and associated proteins form a periodic

$241 \quad$ cytoskeletal structure in axons. Science 339, 452-456 (2013).

242 24. Chaigne, A. et al. A soft cortex is essential for asymmetric spindle positioning in mouse

243 oocytes. Nat. Cell Biol. 15, 958-966 (2013).

$24425 . \quad$ Kumar, R., Saha, S. \& Sinha, B. Cell spread area and traction forces determine myosin-II-

245 based cortex thickness regulation. Biochim. Biophys. Acta BBA - Mol. Cell Res. 1866, 118516

246 (2019).

247 26. Walcott, S. \& Sun, S. X. A mechanical model of actin stress fiber formation and substrate

248 elasticity sensing in adherent cells. Proc. Natl. Acad. Sci. 107, 7757-7762 (2010).

249 27. Vignaud, T. et al. Stress fibres are embedded in a contractile cortical network. Nat.

250 Mater. 1-11 (2020) doi:10.1038/s41563-020-00825-z.

251 28. Mandal, K. et al. Soft hyaluronic gel promotes cell spreading, stress fibers, focal adhesion,

252 membrane tension by phosphoinositide signaling, not traction force. ACS Nano 13, 203-214

253 (2019).

254 29. Shi, Z., Graber, Z. T., Baumgart, T., Stone, H. A. \& Cohen, A. E. Cell membranes resist flow.

255 Cell 175, 1769-1779.e13 (2018). 
256 30. Raucher, D. et al. Phosphatidylinositol 4,5-bisphosphate functions as a second messenger

257 that regulates cytoskeleton-plasma membrane adhesion. Cell 100, 221-228 (2000).

258 31. Pontes, B. et al. Cell cytoskeleton and tether extraction. Biophys. J. 101, 43-52 (2011).

259 32. Pontes, B. et al. Membrane elastic properties and cell function. PLoS One 8, e67708

260 (2013)

261 33. Wallingford, J. B., Liu, K. J. \& Zheng, Y. Xenopus. Curr. Biol. 20, 263-264 (2010).

262 34. Koser, D. E. et al. Mechanosensing is critical for axon growth in the developing brain. Nat.

$263 \quad$ Neurosci. 19, 1592-1598 (2016).

264 35. Nieuwkoop, P. D. \& Faber, J. Normal table of xenopus laevis (daudin). (Routledge, 1994).

265 36. Swift, J. et al. Nuclear lamin-A scales with tissue stiffness and enhances matrix-directed

266 differentiation. Science 341, (2013).

267 37. Moshayedi, P. et al. Mechanosensitivity of astrocytes on optimized polyacrylamide gels

268 analyzed by quantitative morphometry. J. Phys. Condens. Matter 22, (2010).

269 38. Mc Hugh, J., Andresen, K. \& Keyser, U. F. Cation dependent electroosmotic flow in glass

270 nanopores. Appl. Phys. Lett. 115, 113702 (2019).

271 39. Gittes, F. \& Schmidt, C. F. Chapter 8 signals and noise in micromechanical measurements.

272 in Methods in cell biology. vol. 55 129-156 (Academic Press, 1997).

273 40. Hochmuth, R. M., Shao, J. Y., Dai, J. \& Sheetz, M. P. Deformation and flow of membrane

274 into tethers extracted from neuronal growth cones. Biophys. J. 70, 358-369 (1996).

275 41. Moshayedi, P. et al. The relationship between glial cell mechanosensitivity and foreign

276 body reactions in the central nervous system. Biomaterials 35, 3919-3925 (2014). 
bioRxiv preprint doi: https://doi.org/10.1101/2021.11.09.467973; this version posted November 11, 2021. The copyright holder for this preprint (which was not certified by peer review) is the author/funder, who has granted bioRxiv a license to display the preprint in perpetuity. It is made available under aCC-BY-NC 4.0 International license.

277 42. Corder, G. W. \& Foreman, D. I. Nonparametric statistics for non-statisticians: A step-by-

278 step approach. (Wiley-Blackwell, 2009). 
a Peak force: overcoming membrane cortex adhesion locally

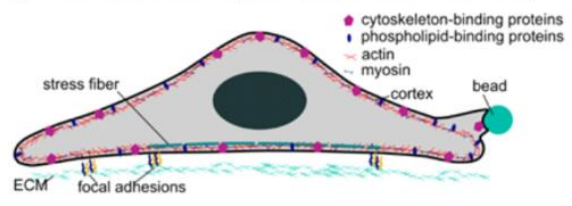

b Steady state force: contributions from adhesion mediating
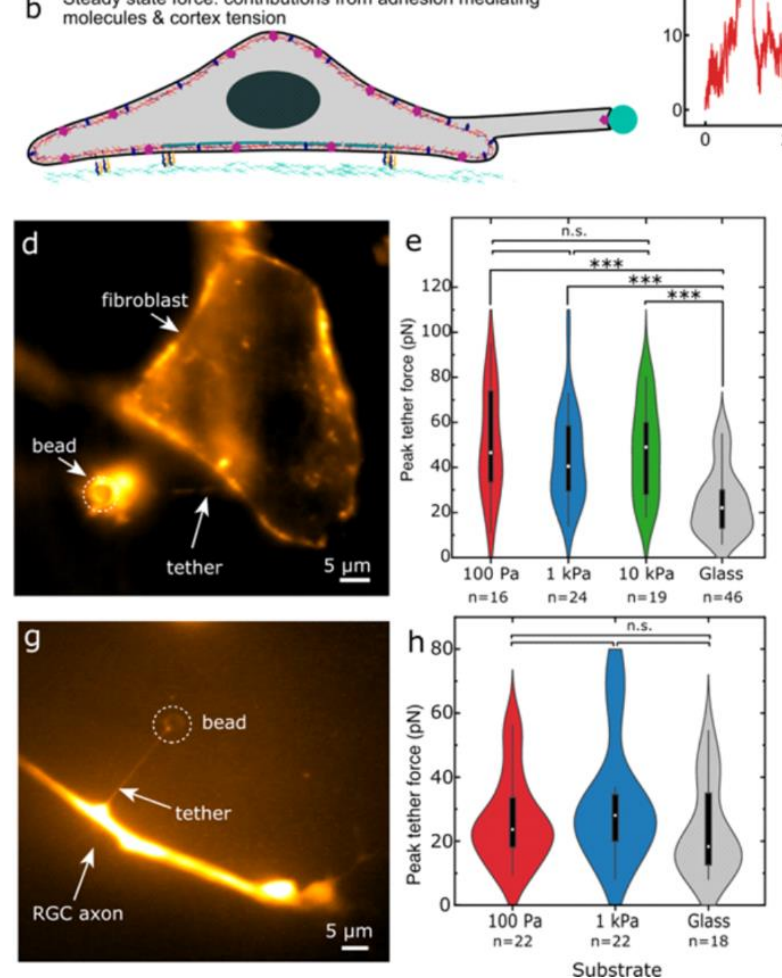
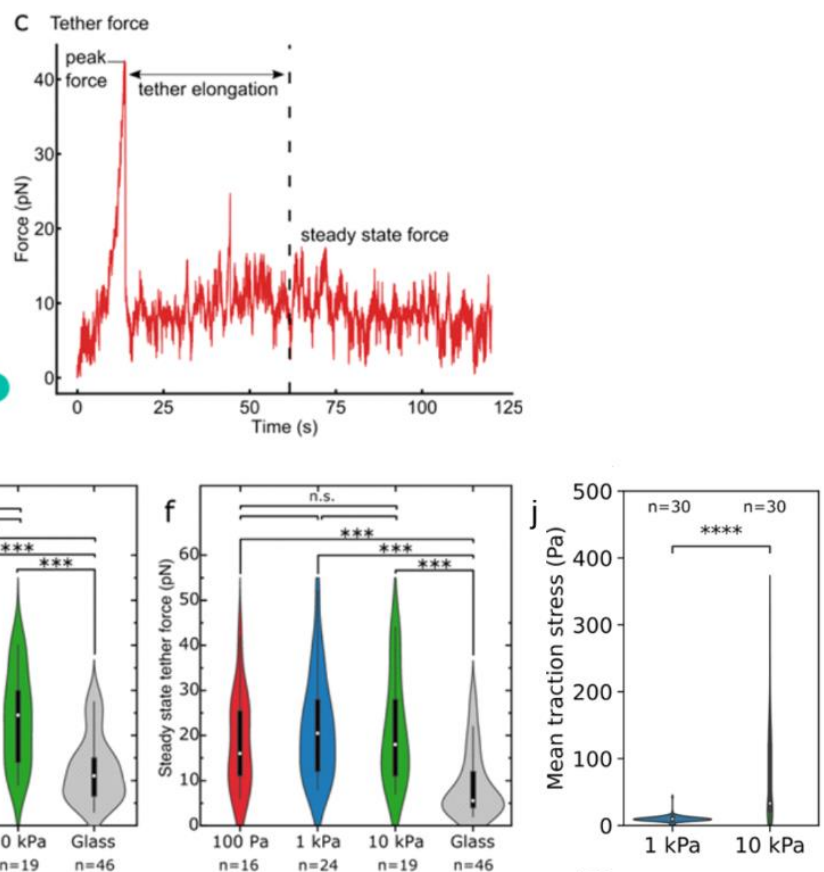

Figure 1: Peak and steady state tether forces provide different insights into cell properties. a) When an

284 OT pull is initiated, the local membrane-cortex adhesion first needs to be overcome, resulting in the

285 peak force. b) After tether extension, the bead is held stationary, and the steady state force, which scales with membrane tension, is recorded.c) A typical force curve recorded during a tether pull shows a peak (see (a)) at the beginning of the pull and a steady state force when the tether is held at its maximum extension (see (b). ( $d-f, j) 3 T 3$ fibroblasts and $(g-I, k)$ Xenopus retinal ganglion cell axons

289 on different substrates; shear moduli are provided in $\mathrm{Pa}$. d) Fluorescence image of a lipid tether pulled

290 from a 3 T3 fibroblast. e) Peak tether forces (PFs) and f) steady state tether forces (SSFs) of fibroblasts 
bioRxiv preprint doi: https://doi.org/10.1101/2021.11.09.467973; this version posted November 11,2021 . The copyright holder for this preprint (which was not certified by peer review) is the author/funder, who has granted bioRxiv a license to display the preprint in perpetuity. It is made available under aCC-BY-NC 4.0 International license.

291 are similar on all hydrogel substrates irrespective of their stiffness and higher compared to on glass. g)

292 Fluorescence image of a lipid tether pulled from an axon. h) PFs in axons are similar on all hydrogel

293 and glass substrates. i) SSFs are similar on all hydrogels and higher if compared to glass. $j$-k) Traction

294 forces measured for (j) 3T3 fibroblasts and ( $k$ ) neuronal axons. Both cell types exerted higher traction

295 forces on stiff hydrogels compared to softer ones.

296 
bioRxiv preprint doi: https://doi.org/10.1101/2021.11.09.467973; this version posted November 11,2021 . The copyright holder for this preprint (which was not certified by peer review) is the author/funder, who has granted bioRxiv a license to display the preprint in perpetuity. It is made available under aCC-BY-NC 4.0 International license.
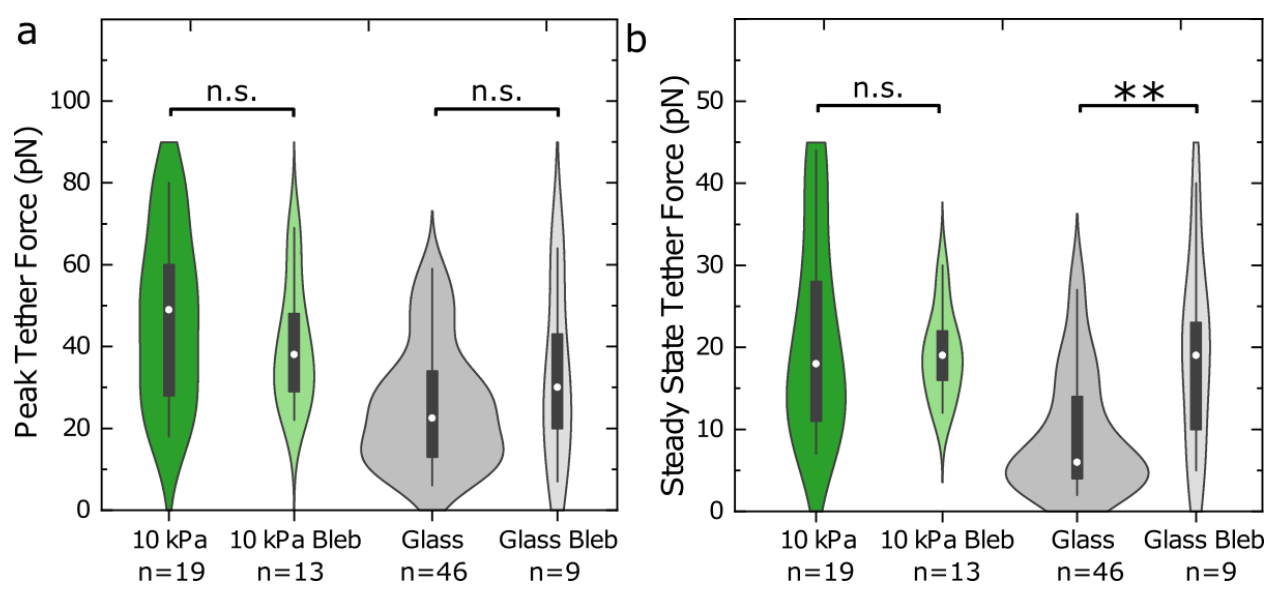

298 Figure 2: The effect of actomyosin contractility on PFs and SSFs in fibroblasts. a) Blebbistatin

299 application did not significantly alter PFs on glass and hydrogel substrates. b) SSFs are unaffected in

300 cells growing on hydrogels but significantly increase on glass substrates after blebbistatin treatment. 
302

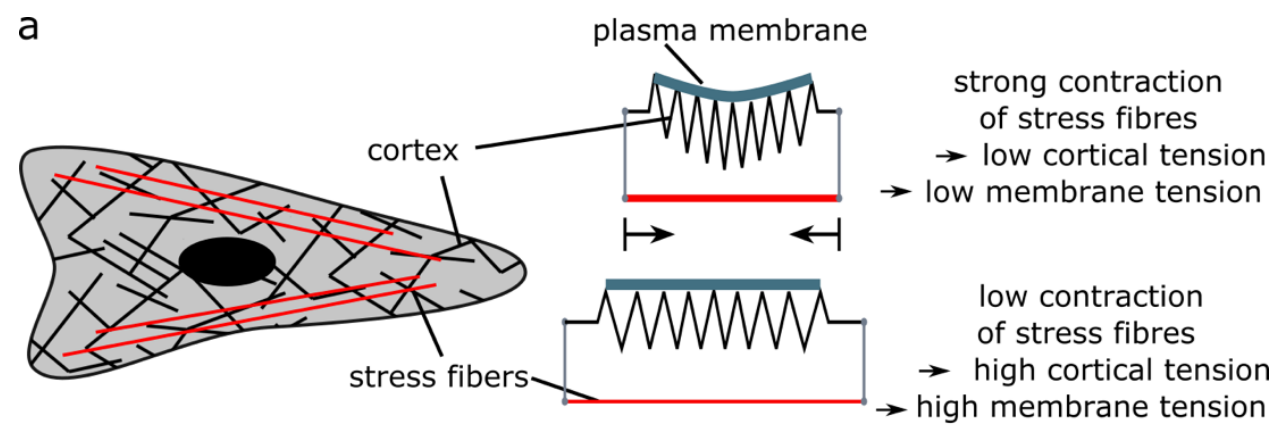

b

axonal structure

303

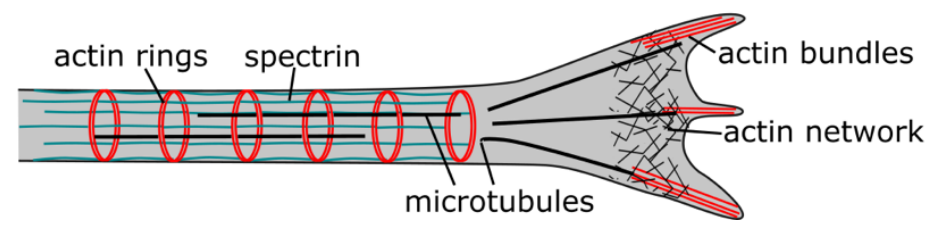

304

305 Figure 3: Toy model of substrate type-dependent effective membrane tension. a) Schematic

306 representation of the cell cortex and stress fibres in fibroblasts. The strong contraction of stress fibres

307 on glass substrates might relax the tension in the cortex and thus reduce membrane tension. The

308 relaxation of these stress fibres, on the other hand, might increase the cortical tension. The top model

309 illustrates the tension in untreated fibroblasts grown on glass. The bottom shows the tension in the

310 relevant components in fibroblasts on glass treated with blebbistatin or in untreated fibroblasts

311 grown on hydrogels. b) The axon has a periodic actin ring-spectrin network underneath its

312 membrane ${ }^{23}$, which is likely rather static and poorly adhered to the membrane. 